\title{
Factor of interfacial potential for the surface plasmon-polariton resonance sensor response
}

\author{
A.M. Lopatynskyi ${ }^{1}$, O.G. Lopatynska ${ }^{2}$, M.D. Guiver ${ }^{3}$, L.V. Poperenko ${ }^{2}$, V.I. Chegel ${ }^{1}$ \\ ${ }^{1}$ V. Lashkaryov Institute of Semiconductor Physics, National Academy of Sciences of Ukraine, \\ 41, prospect Nauky, 03028 Kyiv, Ukraine, +38 (044) 525-56-26, \\ e-mail:lop2000@ukr.net,vche111@yahoo.com \\ ${ }^{2}$ National Taras Shevchenko University of Kyiv, build. 1, 2, prospect Glushkova, \\ 03022 Kyiv, Ukraine, +38 (044) 526-22-96, e-mail: olga_lopatynska@ukr.net,plv@univ.kiev.ua \\ ${ }^{3}$ Institute for Chemical Process and Environmental Technology, National Research Council, \\ 1200 Montreal Road, Ottawa, Ontario, K1A 0R6, Canada, (613) 993-9753, \\ e-mail: Michael.Guiver@nrc-cnrc.gc.ca
}

\begin{abstract}
In this work, we investigate how the application of an external potential difference to the sensitive gold-electrolyte interface influences the optical response of a sensor based on the surface plasmon-polariton resonance (SPPR). The SPPR resonant angle shift was registered for an aqueous solution of sulfuric acid as an electrolyte at different potential sweep rates. From the measurements of SPPR curves versus the applied voltage, the potential of zero charge of the gold electrode in the electrolyte solution was estimated. To explain the external voltage influence on the SPPR sensor response, a theoretical model was used that takes into account three factors: free electron concentration change in the space-charge layer (SCL) in the surface layer of gold, dependence of the capacity of electrical double layer at the interface on the voltage, and gold film surface roughness.
\end{abstract}

Keywords: surface plasmon-polariton resonance, interfacial potential, electrical double layer.

Manuscript received 22.07.08; accepted for publication 20.10.08; published online 11.11.08.

\section{Introduction}

Many modern chemical and biological sensors are based on the surface plasmon-polariton resonance (SPPR) phenomenon [1-6]. The SPPR method is a very sensitive optical technique based on registration of the excitation of surface plasmon-polaritons (surface charge density waves that can be generated on the boundary between the highly-conductive metal and the dielectric and propagate along this boundary) by light $[7,8]$. Using the SPPR method, it is possible to measure optical constants of materials which are located at the sensitive surface of the sensor with high precision. SPPR sensors are widely used for the real-time investigation of liquid systems (e.g. biomolecules in buffer solutions, electrolytes etc.). Measurements using the SPPR sensor are often carried out under the external potential difference applied to the metal-liquid sensor interface [9-13]. The problem arises here in the correct interpretation of the obtained results taking into account the influence of the potential difference both on the object of investigation and on the sensor sensitive part.
Investigation of the influence of potential applied to the metal-electrolyte interface on the optical properties of this interface using surface plasma waves excitation has been carried out actively during recent years. From electroreflectance experiments, it was revealed that the optical properties of metal electrode surface depend significantly on the applied potential [1419]. Later it was shown that the surface plasmonpolariton excitation energy in thin silver and gold films also depended on the applied potential [20]. In this work, the theoretical explanation of this effect was developed only in the approximation of free electron concentration change in the surface layer of the metal upon the potential application. In articles [21,22], excitation of surface plasmon-polaritons at the silver-electrolyte solution interface was investigated and the influence of applied potential on the angular position of SPPR was observed. It was noticed that at a fixed light wavelength, the resonance shifted towards greater values of the surface plasmon-polariton wave vector (greater angles of light incidence) upon increase of the applied potential above the potential of zero charge of the electrode. An 
increase in the SPPR curve half-width was also detected under the above mentioned conditions. In addition, a method to estimate the potential of zero charge of the metal electrode in the electrolyte solution by SPPR measurements was suggested in these references [21, 22]. The gold-electrolyte interface was also investigated using the ellipsometric method of surface plasma waves detection [23]. In the framework of this method, the possibility to separate the influence of applied voltage on the electrons in metal and ions in electrolyte was shown. The penetration depth of static electric field into gold was demonstrated to be not more than $1 \AA$.

Lately, thin (40-45 nm) gold films are even more often used as a bearer of the plasmon-polariton oscillations due to their stability in aqueous solutions and the possibility of easy chemical modification of the gold surface. That is why the investigation and theoretical explanation of external potential difference influence on the optical properties of the gold-electrolyte interface, which the SPPR sensor response is dependent on, is an urgent problem.

\section{Experimental setup}

A two-channel optoelectronic SPPR sensor "Plasmon-5" (ISP NASU, Kyiv) (Fig. 1), based on Kretschmann configuration with semiconductor laser as a $p$-polarized light source $(\lambda=650 \mathrm{~nm})$ was used in the work. A $45 \mathrm{~nm}$ thick gold film produced by a thermal vacuum evaporation method was exploited as a plasmonpolariton oscillations bearer. To enhance the adhesion, a thin chromium layer (up to $5 \mathrm{~nm}$ ) was evaporated onto the glass slide before the evaporation of gold. Thermal evaporation was performed in vacuum $\left(10^{-6} \mathrm{~mm} \mathrm{Hg}\right)$ on the substrates at the room temperature. After evaporation, the films were annealed at $120^{\circ} \mathrm{C}$ for $30 \mathrm{~min}$.

For the simultaneous SPPR and electrochemical measurements, we used classical three-electrode electrochemical cell. An IPC-compact (IPC RAS, Moscow) potentiostat with a gold film as a working electrode and $0.5 \mathrm{~mm} \mathrm{Pt}$ wire and $\mathrm{Ag} / \mathrm{AgCl}$ electrode as counter and reference electrodes, correspondingly, was employed for cyclic voltammetry (Fig. 1). Utilization of the three-electrode system allows stable values to be obtained for the potential difference between the working electrode and electrolyte with respect to the reference electrode, in contrast to the two-electrode system without the reference electrode, which was used in reference [24]. $0.1 \mathrm{M} \mathrm{H}_{2} \mathrm{SO}_{4}$ aqueous solution was used as electrolyte.

\section{Theory}

\subsection{Electrical double layer}

Electrochemical reactions on the interface may progress upon application of an external voltage to the goldelectrolyte system. If the gold electrode potential is sufficient for oxidation or reduction of the electrode, molecules or ions on its interface, then an electrochemical reaction begins, and the current flows through the gold-electrolyte boundary. If the conditions on the interface are such that the electrochemical reactions do not take place, then the current doesn't flow across the interface and the electrical double layer (EDL) (Fig. 2) at the boundary behaves like a capacitor. The potential region where this behaviour is observed is the well-known double layer region.

According to the double layer theory by Stern, the differential capacity of the EDL unit area $C_{E D L}$ equals the capacity of two series-connected capacitors $C_{\mathrm{H}}$ and $C_{\mathrm{GC}}$ that correspond to the compact layer in the Helmholtz theory and the diffuse layer in the GouyChapman theory [25]:

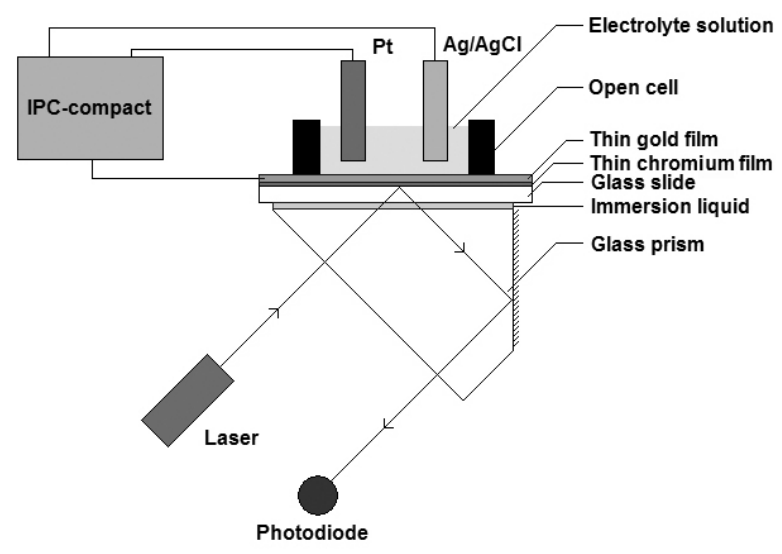

Fig. 1. Simplified scheme of the experimental setup.

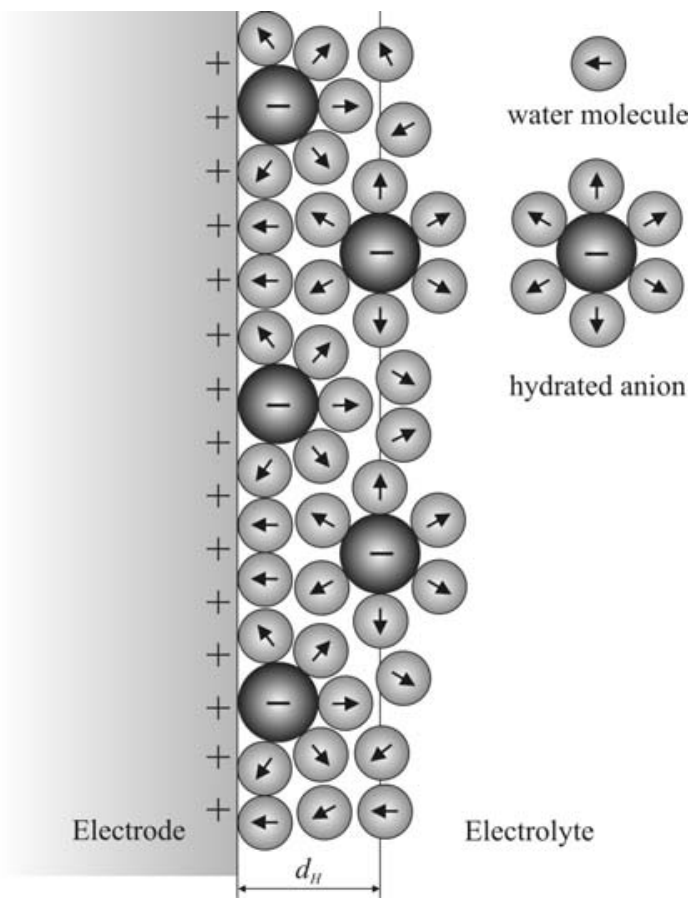

Fig. 2. Model of electrical double layer on the positively charged electrode surface. 


$$
\begin{aligned}
& \frac{1}{C_{E D L}}=\frac{1}{C_{\mathrm{H}}}+\frac{1}{C_{\mathrm{GC}}}=\frac{d_{\mathrm{H}}}{\varepsilon \varepsilon_{0}}+ \\
& +\frac{1}{\left(2 \varepsilon \varepsilon_{0} z^{2} e_{0}^{2} N_{\mathrm{A}} \cdot 1000 c / k T\right)^{1 / 2} \cosh \left(z e_{0} U / 2 k T\right)},
\end{aligned}
$$

where $d_{\mathrm{H}}$ is the compact layer thickness, $\varepsilon$ is the static dielectric permittivity of the electrolyte, $\varepsilon_{0}$ is the vacuum dielectric permittivity, $\mathrm{z}$ is the ion charge, $e_{0}$ is the electron charge, $N_{\mathrm{A}}$ is the Avogadro constant, $c$ is the electrolyte concentration in $\mathrm{mol} / \mathrm{l}, \quad k$ is the Boltzmann constant, $T$ is the electrolyte temperature and $U$ is the potential at the distance $d_{\mathrm{H}}$ from the metal surface. Potential $U$ is determined from the equation (2) [26]:

$U_{0}=U+\sqrt{\frac{8 k T \cdot 1000 N_{\mathrm{A}} c}{\varepsilon \varepsilon_{0}}} \sinh \left(\frac{z e_{0} U}{2 k T}\right) \cdot d_{\mathrm{H}}$,

where $U_{0}$ is the total potential difference between the metal electrode and electrolyte. Then the surface charge density $\Delta \sigma$ that appears on the electrode surface is represented by the equation (3) [25]:

$$
\Delta \sigma=\int_{U_{p z c}}^{U_{0}} C_{E D L} d U
$$

where $U_{p z c}$ is the potential of zero charge of the electrode in the present electrolyte.

Formation of a charge on the gold electrode surface during the charging of EDL leads to a change of the free electron concentration in the surface layer of gold, which is one of the effects taking place when applying the voltage to a gold-electrolyte system. As the static electric field penetrates the metal to the distance $d \sim 1 \AA$, which corresponds to the thickness of the space-charge layer (SCL) near the surface of gold, then the change of the free electron concentration, which is defined by the equation (4), takes place in this thin layer only [20]:

$\Delta N=\frac{\Delta \sigma}{d \cdot e_{0}}$.

This results in the change of the free electron contribution to the relative dielectric permittivity of this layer, which is represented by the equation (5) [20]:

$\Delta \varepsilon_{m}=\left(1-\varepsilon_{m}^{f}\right) \frac{\Delta N}{N}$,

where $\varepsilon_{m}^{f}$ is the free electron contribution to the relative dielectric permittivity of the metal and $N$ is the free electron concentration in the metal. The free electron contribution to the relative dielectric permittivity of the metal is defined by the equation (6) [27]:

$\varepsilon_{m}^{f}=1-\frac{\omega_{p}^{2}}{\omega^{2}-i \frac{\omega}{\tau}}$, where $\omega_{p}=\sqrt{\frac{N e_{0}^{2}}{\varepsilon_{0} m}}$ is the plasma frequency, $m$ is the mass of an electron, $\omega$ is the light angular frequency, $\tau$ is the free electron relaxation time.

\subsection{Calculation of the SPPR sensor response}

Calculation of the SPPR sensor optical response (angular position of the SPPR) on the application of the electrical voltage to the gold-electrolyte interface was carried out using the scattering matrix formalism [28] for the multilayer system (Fig. 3).

The scattering matrix $S$ connects complex strengths of electric fields on the first and the last boundaries of the multilayer structure. It can be represented in equation (7) as a product of the interface matrices $I$ and the layer matrices $L$ that describe the influence of the separate interfaces and layers in the multilayer structure:

$S=I_{01} L_{1} I_{12} L_{2} \ldots L_{m} I_{m(m+1)}$.

Interface and layer matrices are shown in equation (8):

$$
\begin{aligned}
& I_{j(j+1)}=\left(1 / t_{j(j+1)}\right)\left[\begin{array}{cc}
1 & r_{j(j+1)} \\
r_{j(j+1)} & 1
\end{array}\right], \\
& L_{j}=\left[\begin{array}{cc}
e^{i \beta_{j}} & 0 \\
0 & e^{-i \beta_{j}}
\end{array}\right],
\end{aligned}
$$

where $t_{j(j+1)}$ and $r_{j(j+1)}$ are Fresnel amplitude coefficients of transmission and reflection of $p$-polarized light for the $j(j+1)$ boundary, $\beta_{j}=\frac{2 \pi d_{j} \tilde{N}_{j}}{\lambda} \cos \theta_{j}$ is the phase thickness of the $j$-th layer, $d_{j}$ is the thickness of the $j$-th layer, $\tilde{N}_{j}$ is the complex refractive index of the $j$-th layer, $\theta_{j}$ is the angle between the light propagation direction and the perpendicular to the boundary in the $j$-th layer. Fresnel amplitude coefficients of transmission and reflection of $p$-polarized light for the $j(j+1)$ boundary are defined by the following equations (9) [29]:

$$
\begin{aligned}
t_{j(j+1)} & =\frac{2 \widetilde{N}_{j} \cos \theta_{j}}{\widetilde{N}_{j+1} \cos \theta_{j}+\widetilde{N}_{j} \cos \theta_{j+1}}, \\
r_{j(j+1)} & =\frac{\widetilde{N}_{j+1} \cos \theta_{j}-\widetilde{N}_{j} \cos \theta_{j+1}}{\widetilde{N}_{j+1} \cos \theta_{j}+\widetilde{N}_{j} \cos \theta_{j+1}} .
\end{aligned}
$$

When the scattering matrix for the multilayer system is calculated, it is possible to determine the energetic coefficient of reflection of $p$-polarized light $(R)$ from the multilayer structure using the equation (10):

$R=\left(\frac{S_{21}}{S_{11}}\right)^{2}$ 


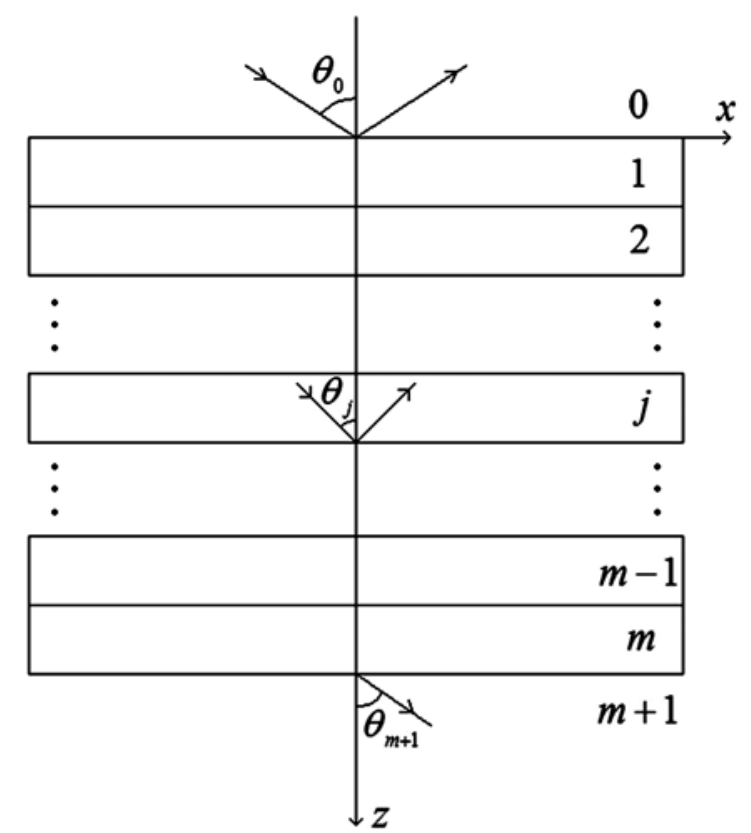

Fig. 3. Schematic drawing of the multilayer structure and the propagation of light through it.

where the indices indicate the corresponding elements of the matrix $S$. Using the calculated dependences $R\left(\theta_{0}\right)$ for the investigated multilayer system at $\theta_{0}>\theta_{T I R}\left(\theta_{T I R}\right.$ is the limiting angle of the total internal reflection) it is possible to determine their minimum angular position $\theta_{S P P R}$ that will correspond to the rise of the SPPR.

\subsection{Calculation of the complex refractive index of the surface layer of the gold film}

The gold film produced by the thermal vacuum evaporation method is characterized by a rough surface; therefore, this has to be taken into account for the theoretical calculation of the optical response. In the present research, the surface layer of gold is modeled as a statistical heterosystem that consists of 3 components: gold surface, SCL in gold with thickness of $1 \AA$, whose optical properties are dependent on the applied external voltage, and electrolyte. Since volume fractions of the components are comparable, the symmetrical Bruggeman approximation for the statistical heterosystem is used to calculate the effective complex refractive index $\widetilde{N}$ for this heterosystem using the equation (11) [30]:

$\sum_{i} f_{i} \frac{N_{i}^{2}-\widetilde{N}^{2}}{N_{i}^{2}+2 \widetilde{N}^{2}}=0$

where $i=1,2,3$ is the sequence number of the component, $f_{i}$ and $N_{i}$ are the volume fractions and refractive indices for the corresponding components of the heterosystem.

\section{Results and discussion}

As a result of simultaneous SPPR and electrochemical measurements cyclic voltammograms of the goldelectrolyte interface (Fig. 4a) and temporal dependences of the angular position of SPPR curves minima (Fig. 4b) were obtained.

Typical features of the electrochemical processes are clearly visible on the cyclic voltammogram shown in Fig. 4a. During the change of the potential from -0.6 to $1.4 \mathrm{~V}$ at the potential difference greater than $0.7 \mathrm{~V}$, the electrochemical reaction of gold surface oxidation takes place $[13,31]$ :

$\mathrm{Au}+2 \mathrm{H}_{2} \mathrm{O} \rightarrow \mathrm{AuOH} \cdot \mathrm{H}_{2} \mathrm{O}+\mathrm{H}^{+}+\mathrm{e}^{-}$,

$\mathrm{AuOH} \rightarrow \mathrm{AuO}+\mathrm{H}^{+}+\mathrm{e}^{-}$.
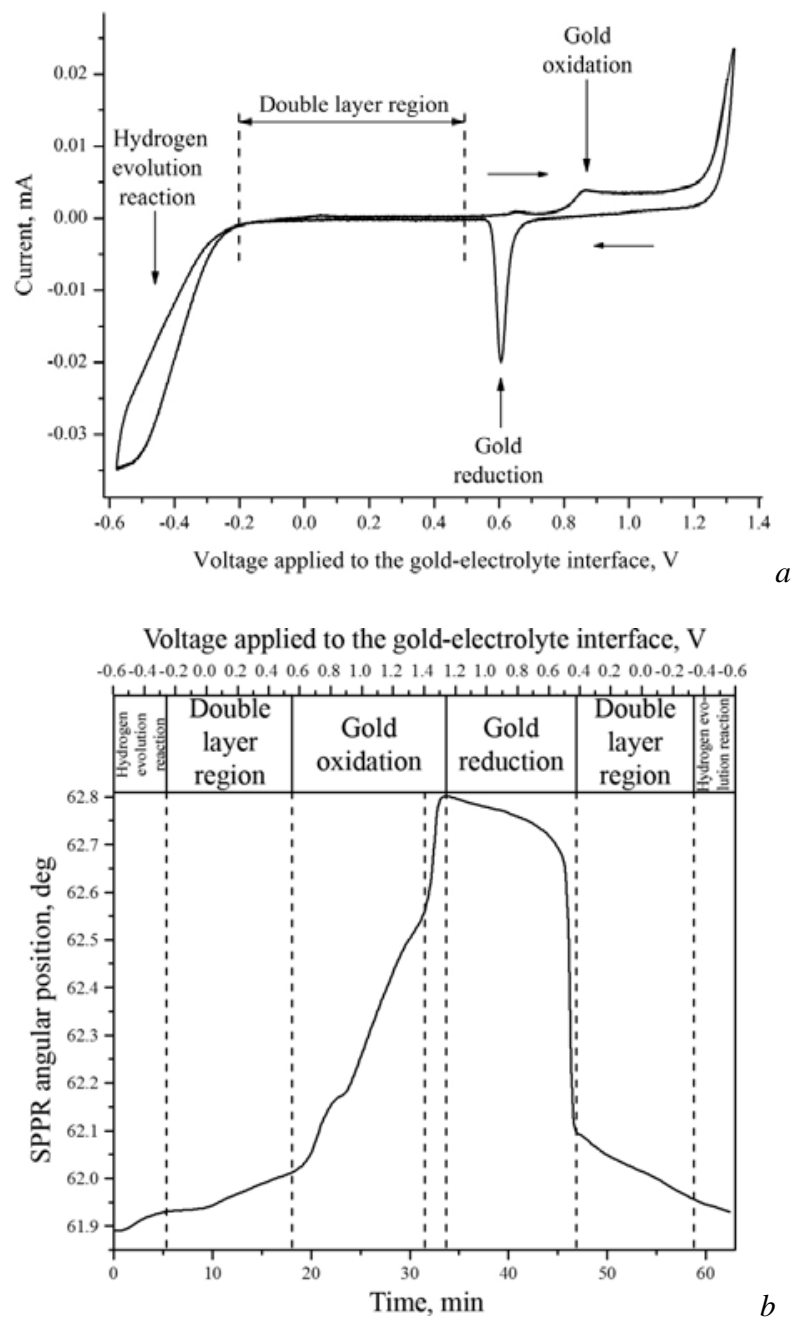

Fig. 4. a) Measured cyclic voltammogram of the gold-sulfuric acid solution interface. Potential difference between gold and electrolyte changed in the region $(-0.6 \rightarrow+1.4 \rightarrow-0.6) \mathrm{V}$ with the sweep rate of $0.001 \mathrm{~V} / \mathrm{s}$. Potential change direction is indicated by the arrows. All potentials are reported with respect to the reference electrode. b) Measured temporal dependence of the SPPR curves minimum angular position (sensogram). Dash line in the center indicates the moment when potential starts to change in the opposite direction. 
This leads to the formation of a thin gold oxide layer on the gold electrode surface that results in an increase in the SPPR angle (Fig. 4). During the course of change of the potential backwards from 1.4 to $-0.6 \mathrm{~V}$ at the potential difference about $0.7 \mathrm{~V}$, the electrochemical reaction of the gold surface reduction begins, which is opposite to the above mentioned process. This leads to the dissolution of the oxide film on the surface and to a decrease in the SPPR angle (Fig. 4). At a potential difference lower than $-0.3 \mathrm{~V}$, the hydrogen evolution reaction begins on the gold electrode [32]:

$\mathrm{H}^{+}+\mathrm{e}^{-} \rightarrow \frac{1}{2} \mathrm{H}_{2}$ (gas).

The most important potential difference region for the practical experiments is in the region where electrochemical reactions involving substances that the sensor is composed of are absent (double layer region) (Fig. 4). For the investigated system, this region ranges from about -0.2 to $0.5 \mathrm{~V}$. Therefore, the individual measurements in the potential difference region from -0.2 to $0.5 \mathrm{~V}$ were performed, and their results are presented in Fig. 5.
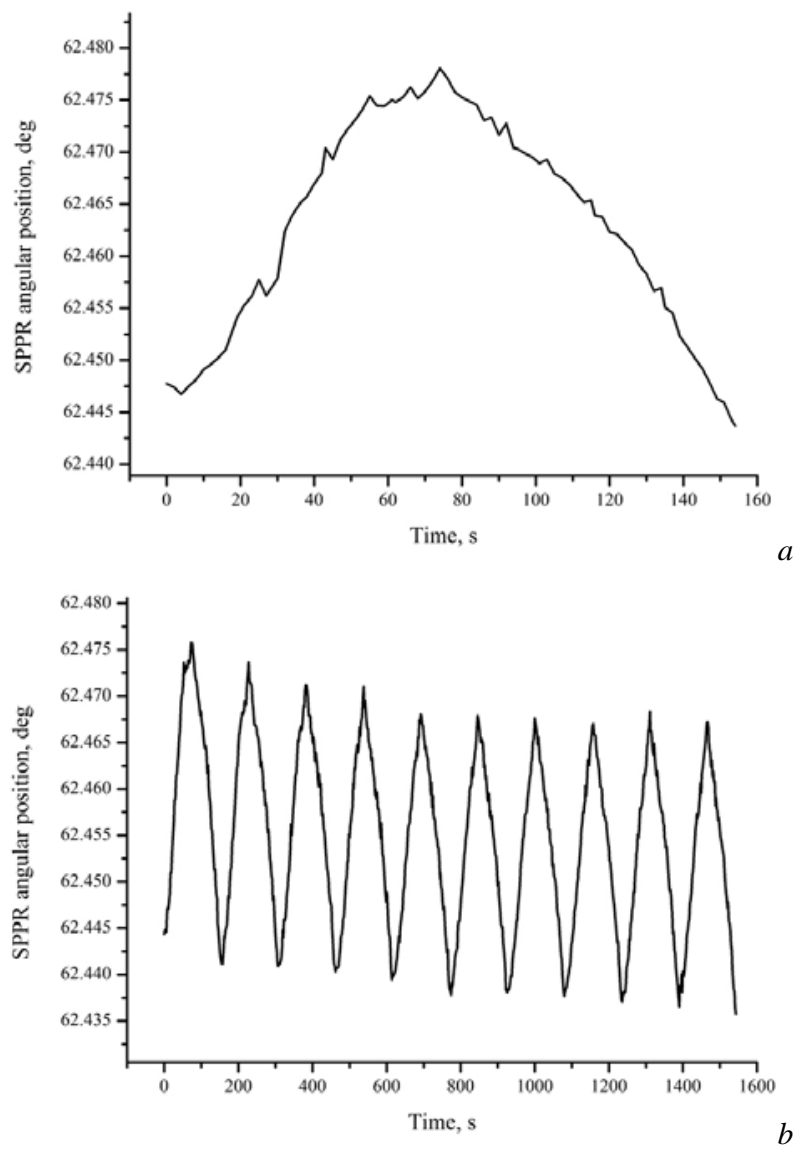

Fig. 5. Measured temporal dependence of the SPPR curves minimum angular position (sensogram) upon the application of the potential difference in the region $(-0.2 \rightarrow+0.5 \rightarrow-0.2) \mathrm{V}$ with the sweep rate of $0.009 \mathrm{~V} / \mathrm{s}$ to the gold-electrolyte interface: a) 1 cycle of the potential change; b) 10 cycles of the potential change.
It was observed that the shift of SPPR angular position increased while decreasing the potential sweep rate when the applied potential difference changed from -0.2 to $0.5 \mathrm{~V}$. This can be caused by slow diffusion processes of "heavy" $\mathrm{SO}_{4}{ }^{2-}$ ions in the electrolyte solution under the influence of the electric field between the electrodes and by following accumulation of these ions at the gold surface. Taking this into account, for the exclusion of above mentioned processes the measurements in the potential difference range from -0.2 to $0.5 \mathrm{~V}$ were performed with $0.009 \mathrm{~V} / \mathrm{s}$ rate. This sweep rate allowed us to record the quantity of SPPR angular position points that was adequate for analysis but minimized the influence of the ion diffusion processes. The sensogram represented in Fig. 5b shows that the processes leading to the SPPR angular position change due to external voltage application are rather reversed at the chosen potential sweep rate. This means that during changing the potential difference in the chosen region the oxide film formation at the gold surface as a result of electrochemical reactions does not occur, which typical hysteresis of the SPPR angle is peculiar to upon repeating the potential change cycles [24].

The study of the experimental SPPR response in the double layer region allows us to estimate the potential of zero charge value of the gold electrode in the electrolyte solution [21,22]. Analysis of the dependences of SPPR curves half-widths on the voltage between the gold electrode and electrolyte gives the potential of zero charge value that equals $U_{p z c}=0.17 \pm 0.04 \mathrm{~V}$ versus the used reference electrode.

Theoretical modeling the SPPR curves for the potential difference in the double layer region was carried out for the multilayer system consisting of a semi-infinite glass layer (refractive index $n=1.616$ ), a $5 \mathrm{~nm}$ thick chromium layer $(n=3.66+4.365 \mathrm{i}[33])$, a $45 \mathrm{~nm}$ thick gold layer $(n=0.3+3.55 \mathrm{i}$ [34]), a surface gold layer whose refractive index is calculated using equation (11), and a semi-infinite electrolyte layer $(n=1.3313)$. The surface gold layer parameters calculation was performed using the results of atomic force microscopy (AFM) (Fig. 6) of gold film surface before the immersion in the electrolyte solution. Namely, volume fractions of gold surface, SCL in gold with $1 \AA$ thickness and electrolyte were determined and found to be $0.401,0.103$ and 0.496 , respectively. From the AFM investigation results, the surface gold layer effective thickness was also obtained, which was estimated as equal to the root mean square deviation of height for the rough gold surface, which is $9.8 \AA$.

The following parameters of electrical double layer were used during modeling: compact layer thickness $d_{\mathrm{H}}=8.5 \AA$ (calculated according to the water molecule [35] and the $\mathrm{SO}_{4}{ }^{2-}$ anion [36] sizes), static dielectric permittivity of the dilute sulfuric acid solution $\varepsilon=80$ [33], electrolyte concentration $c=0.1 \mathrm{~mol} / 1$. The 


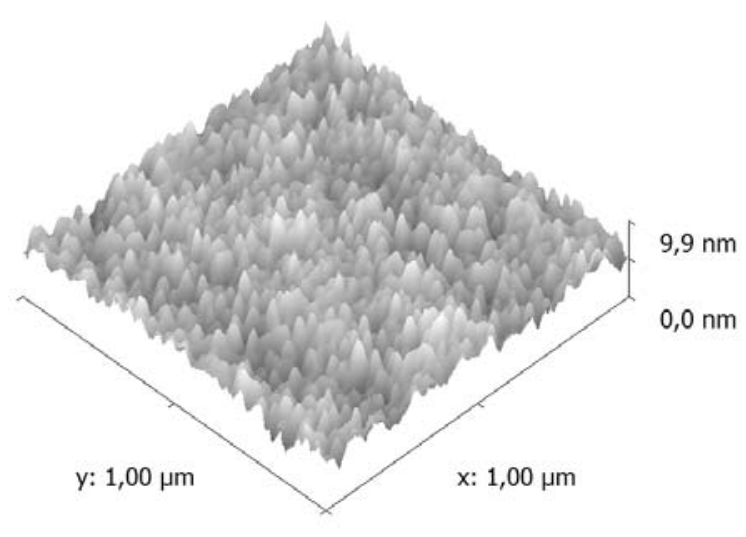

Fig. 6. AFM image of the gold film rough surface.

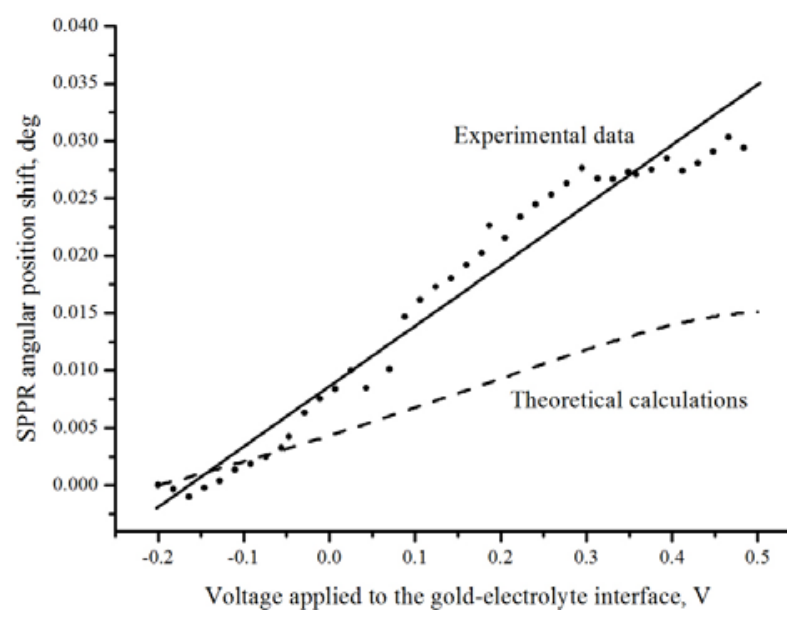

Fig. 7. Theoretically calculated dependence of the angular position shift observed for the minimum of the reflected $p$ polarized light intensity and experimentally obtained dependence of the SPPR angular position shift on the applied potential difference.

following bulk gold parameters were also used for calculations: $\quad \omega_{p}=1.37 \cdot 10^{16} \mathrm{~Hz} \quad[37], \quad \tau=$ $9.3 \cdot 10^{-15} \mathrm{~s}[38], N=5.9 \cdot 10^{28} \mathrm{~m}^{-3}$ [37].

The calculated dependence of SPPR angle shift upon changing the voltage between gold and electrolyte in the range from -0.2 to $0.5 \mathrm{~V}$ for the wavelength of light $\lambda=650 \mathrm{~nm}$ and corresponding experimentally obtained dependence are presented in Fig. 7.

It is apparent from the experimental results that potential difference change between gold electrode and electrolyte from -0.2 to $0.5 \mathrm{~V}$ versus reference electrode causes angular shift of the SPPR curve minimum that is about $0.03^{\circ}$. Theoretical calculations give the value of corresponding SPPR angular position shift, which equals to $0.0151^{\circ}$. The difference between obtained results can be explained by the abovementioned dependence of SPPR angle shift on the potential sweep rate. Probably, diffusion processes still have a noticeable influence on the SPPR sensor response at the chosen potential sweep rate of $0.009 \mathrm{~V} / \mathrm{s}$. Support of high potential sweep rates and faster registration of the SPPR angular position, respectively, will be the aim of further research. More precise calculation of the SCL thickness and the electron concentration distribution [18] would also result in the better theoretical approximation to the experimental results. The obtained result also points to the fact that free electron concentration change in the gold surface layer is not the only mechanism of the electric voltage application influence on the goldelectrolyte interface properties. In particular, optical properties of this interface can depend on the change of electrolyte surface layer properties due to adsorption of ions from solution on the gold surface, and also on adsorbed ions influence on the dielectric permittivity of the surface gold layer due to possible formation of the weak chemical bonds.

\section{Conclusion}

In this work, the influence of application of external potential difference to the gold-electrolyte interface of the SPPR sensor on the optical properties of this interface was investigated and a theoretical explanation of this influence was developed. The potential of zero charge for the gold electrode in $0.1 \mathrm{M}$ sulfuric acid aqueous solution versus the $\mathrm{Ag} / \mathrm{AgCl}$ reference electrode was evaluated and found to be $0.17 \pm 0.04 \mathrm{~V}$. The influence of the applied potential difference value on the shift of SPPR angle position was theoretically calculated taking into account the potential influence on the free electron concentration in the SCL in the surface layer of gold, the dependence of capacity of electrical double layer at the gold-electrolyte interface on the potential difference in the Stern model and the presence of roughness on the gold film surface. It was revealed that the shift of SPPR angular position increases while the potential sweep rate decreases. The presented theoretical approach can be used for the partial correction of the SPPR-sensor response upon the application of the external voltage to the sensor interface. Revealed discrepancies between the theoretical calculations and the experimental results require more accurate calculation of the metal optical constants in the spacecharge layer and taking into account the influence of adsorbed ions on the dielectric permittivity of the gold surface layer under the application of the external potential difference.

\section{Acknowledgements}

This work was supported by NATO CLG grant PDD(CP)-(CBP.NUKR.CLG981776). 


\section{References}

1. C.C. Jung, S.B. Saban, S.S. Yee, R.B. Darling, Chemical electrode surface plasmon resonance sensor // Sensors and Actuators B 32(2), p. 143-147 (1996).

2. M.J. Jory, G.W. Bradberry, P.S. Cann, J.R. Sambles, Surface-plasmon opto-electrochemistry // Sensors and Actuators B 35(1), p. 197-201 (1996).

3. Y. Iwasaki, T. Horiuchi, M. Morita, O. Niwa, Analysis of electrochemical processes using surface plasmon resonance // Sensors and Actuators B 50(2), p. 145-148 (1998).

4. R.J. Green, R.A. Frazier, K.M. Shakesheff, M.C. Davies, C.J. Roberts, S.J.B. Tendler, Surface plasmon resonance analysis of dynamic biological interactions with biomaterials // Biomaterials 21(18), p. 1823-1835 (2000).

5. J. Homola, Present and future of surface plasmon resonance biosensors // Anal. Bioanal. Chem. 377(3), p. 528-539 (2003).

6. M. Malmqvist, Biospecific interaction analysis using biosensor technology // Nature 361, p. 186187 (1993).

7. A. Otto, Excitation of nonradiative surface plasma waves in silver by the method of frustrated total reflection // Z. Phys. 216(4), p. 398-410 (1968).

8. N.L. Dmitruk, V.G. Lytovchenko, V.L. Strygevskyj, Surface Polaritons in Semiconductors and Dielectrics. Naukova dumka, Kyiv, 1989 (in Russian).

9. O.A. Raitman, E. Katz, I. Willner, V.I. Chegel, G.V. Popova, Photonic transduction of a three-state electronic memory and of electrochemical sensing of NADH using surface plasmon resonance spectroscopy // Angewandte Chemie International Edition 40(19), p. 3649-3652 (2001).

10. V. Chegel, O. Raitman, E. Katz, R. Gabai, I.Willner, Photonic transduction of electrochemically-triggered redox-functions of polyaniline films using surface plasmon resonance spectroscopy // Chem. Communs, p. 883-884 (2001).

11. R. Gabai, N. Sallacan, V. Chegel, T. Bourenko, E. Katz, I. Willner, Characterization of the swelling of acrylamidophenylboronic acid - acrylamide hydrogels upon interaction with glucose by Faradaic impedance spectroscopy, chronopotentiometry, quartz-crystal microbalance (QCM) and surface plasmon resonance (SPR) experiments // J. Phys. Chem. B 105(34), p. 8196-8202 (2001).

12. V.I. Chegel, O.A. Raitman, O. Lioubashevski, Yu. Shirshov, E. Katz, I. Willner, Redox-switching of electrorefractive, electrochromic and conducting functions of $\mathrm{Cu}^{2+}$ /polyacrylic acid films associated with electrodes // Advanced Materials 14(21), p. 1549-1553 (2002).

13. V. Chegel, The electrochemically induced processes at the gold-polyaniline film interface: surface plasmon resonance study // Functional Materials 12(2), p. 355-361 (2005).

14. J.D.E. McIntyre, Electrochemical modulation spectroscopy // Surf. Sci. 37, p. 658-682 (1973).

15. J.D.E. McIntyre, W.F. Peck Jr., Optical spectroscopy of adsorbed intermediates in electrochemical reactions. Part 1. - Surface field effects // Faraday Discussions of the Chemical Society 56, p. 122-137 (1973).

16. W.J. Anderson, W.N. Hansen, Electroreflectance and conductance // J. Electroanalyt. Chem. 47(2), p. 229-243 (1973).

17. V.A. Tyagai, O.V. Snitko, Electroreflectance of Light in Semiconductor. Naukova dumka, Kiev, 1980 (in Russian).

18. N.L. Dmitruk and V.A. Tyagai, Theory of plasma electroreflectance of light from semiconductors // Physica status solidi (b) 43(2), p. 557-562 (1971).

19. N.A. Savost'yanova and V.B. Sandomirsky, Light and field control of light in polariton-active metalsemiconductor structures: interface polariton optoelectronics // Semiconductor Science and Technology 8, p. 73-79 (1993).

20. R. Kötz, D.M. Kolb, J.K. Sass, Electron density effects in surface plasmon excitation on silver and gold electrodes // Surf. Sci. 69, p. 359-364 (1977).

21. J.G. Gordon II, S. Ernst, Surface plasmons as a probe of the electrochemical interface // Surf. Sci. 101, p. 499-506 (1980).

22. A. Tadjeddine, Influence of the electrical polarization on the surface plasmon dispersion at metal-electrolyte interfaces // Electrochimica Acta 34(1), p. 29-33 (1989).

23. F. Abelès, T. Lopez-Rios, A. Tadjeddine, Investigation of the metal-electrolyte interface using surface plasma waves with ellipsometric detection // Solid State Communs 16(7), p. 843-847 (1975).

24. V. Lioubimov, A. Kolomenskii, A. Mershin, D.V. Nanopoulos and H.A. Schuessler, Effect of varying electric potential on surface-plasmon resonance sensing // Appl. Opt. 43(17), p. 3426-3432 (2004).

25. Ch. Brett, A. Brett, Electrochemistry. Principles, Methods, and Applications. Oxford University Press, Oxford, 1994.

26. A.J. Bard, L.R. Faulkner, Electrochemical Methods: Fundamentals and Applications. Second ed. John Wiley \& Sons, Inc., New York, 2001.

27. M. Dressel, G. Grüner, Electrodynamics of Solids. Optical Properties of Electrons in Matter. Cambridge University Press, Cambridge, 2002.

28. R.M.A. Azzam, N.M. Bashara, Ellipsometry and Polarized Light. Mir, Moscow, 1981 (in Russian).

29. M. Born, E. Wolf, Principles of Optics, Second ed. Nauka, Moscow, 1973 (in Russian).

30. E.F. Venger, A.V. Goncharenko, M.L. Dmitruk, Optics of Small Particles and Disperse Media. Naukova dumka, Kiev, 1999 (in Ukrainian). 
31. J.E. Garland, K.A. Assiongbon, C.M. Pettit, D.Roy, Surface plasmon resonance transients at an electrochemical interface: time resolved measurements using a bicell photodiode // Analytica Chimica Acta 475(1-2), p. 47-58 (2003).

32. R.R. Salem, The Theory of Double Layer. Fizmatlit, Moscow, 2003 (in Russian).

33. R.D. Lide, Handbook of Chemistry and Physics, 84th ed. CRC Press, London, 2004.

34. N.L. Dmitruk, O.V. Fursenko, O.S. Kondratenko, V.R. Romanyuk, Optical characterization of thin Au films by standard and polaritonic ellipsometry // Semiconductor Physics, Quantum Electronics \& Optoelectronics 6(3), p. 349-353 (2003).
35. Modern Aspects of Electrochemistry No. 31, Eds. J.O’M. Bockris, R.E. White, B.E. Conway. Kluwer Academic Publishers, New York, 1999.

36. G.A. Krestov, Thermodynamics of the Ionic Processes in Solutions. Second ed. Khimiya, Leningrad, 1984 (in Russian).

37. M. Fox, Optical Properties of Solids. Oxford University Press, Oxford, 2001.

38. P.B. Johnson, R.W. Christy, Optical constants of the noble metals // Phys. Rev. B 6(12), p. 43704379 (1972). 